\title{
New Powerful Methods for Photometry of CCD Images in Crowded Fields
}

\author{
C. Alard \\ Institut d'Astrophysique de Paris, 98 bis boulevard Arago, 75014 Paris \\ France
}

\begin{abstract}
Image subtraction is an interesting new alternative to the classical profile fitting method (DAOPHOT or DoPHOT) for finding variable stars and producing their light curves. In crowded fields this new method can lead to large improvements in the photometric accuracy. The method is based on finding the best kernel solution in order to match two images as closely as possible. This approach leads to simple mathematical equations. It is possible to find a general solution to these equations which require very reasonable computing times. It is also shown that even in the case of a spatially variable kernel an optimal solution can be found with minimum computing time. Constant flux scaling can be imposed in this case without changing the basis of the algorithm. The method is illustrated using a set of images of the central region of the globular cluster M5. Only 26 variables were found by processing this data set with DoPHOT, while 61 were found with image subtraction. A large photometric improvement was also found for the 26 variables in common. The maximum improvement achieved by using image subtraction was a factor of 20 with respect to DoPHOT. The accuracy achieved with image subtraction is comparable to what was achieved with HST in a small region around the M5 center. One consequence of this photometric improvement was the discovery of an RR Lyrae star pulsating in a nonradial mode in M5. Finally, it is concluded that image subtraction is a technique of choice when dealing with variability, and that it is important to use it when the field is crowded. It is also important to note that image subtraction may open new possibilities in the investigation of very crowded fields, even with relatively small telescope from the ground.
\end{abstract}

\section{Introduction}

Image subtraction is a new general method that can be used to find variable stars and produce their light curves. This new method is particularly efficient in crowded stellar fields or in fields with small-scale variation of the sky background. The fundamental difference between this method and the classical profile fitting methods (DoPHOT; Schechter, Mateo, \& Saha 1993; DAOPHOT: Stetson 1987) is that image subtraction is purely differential. DAOPHOT or DoPHOT attempt to make an absolute exhaustive model of the image by using a sum of point spread functions (PSF). However, in crowded stellar fields, the number of stars is so large that at the image resolution only a small fraction of the stars are resolved 


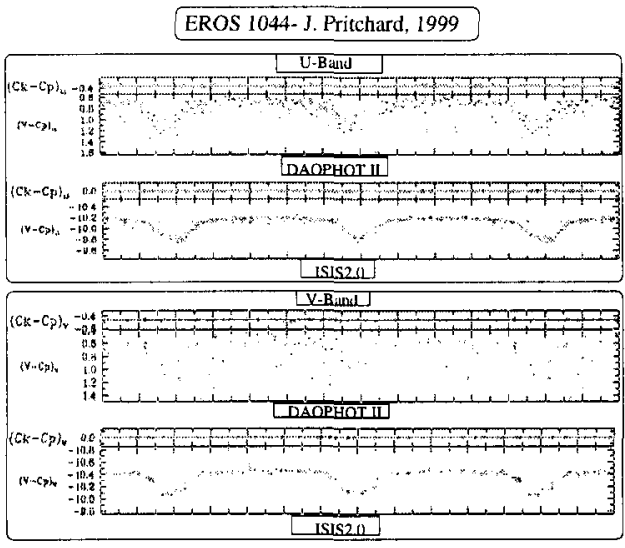

Figure 1. Comparison between image subtraction (ISIS) and DAOPHOT II. The variable star EROS-1044 is situated in a very dense region in the bar of the LMC. The large difference of quality between image subtraction and DAOPHOT is due to the crowding of the field. This case is a good illustration of the ability of image subtraction to achieve almost optimal results, even in case of substantial blending of the stellar fluxes.

and actually seen. In such conditions it is obvious that a full absolute model of the image will fail, and that DoPHOT and DAOPHOT will never produce an optimal result (although good results can be obtained for bright objects). Similar problems will occur in fields containing diffuse objects which cause large variations of the sky background at small scale. Since making a model of the diffuse objects is quite impossible we are in the same situation as in crowded fields. We see that solving the full image problem in the case we described is hopeless, and, since we are interested only in studying the variations in the image, a purely differential method like image subtraction should be more prone to give optimal results. We will even see that within the framework of image subtraction the problem can be expressed in simple mathematical equations for which optimal solutions can be derived.

\section{Comparison of Image Subtraction with Classical Methods}

The light curve of the variable star EROS-1044 was made by Pritchard (1999) using both DAOPHOT II and the ISIS image subtraction package (Alard 1999a). This variable is situated in a very crowded field in the bar of the LMC. Due also to large pixels and not very sharp images, EROS-1044 is very blended. In such a case a large improvement is expected by using image subtraction. This improvement is clearly visible in Fig. 1; the image subtraction photometry is several times better than the DAOPHOT II photometry. Similar large improvements were also found by Alard (1999b) when comparing image subtraction photome try and DoPHOT photometry of the OGLE microlensing events. 


\section{Method}

The basic idea of image subtraction is: provided we can transform 2 images to exactly the same seeing (or said differently to the same PSF), they should subtract perfectly, except around the variable objects. Of course, flux scaling and differential background subtraction of the images is also necessary to achieve perfect subtraction of the image. It is easy to understand that imaging in astronomy is essentially a blurring process, by the atmosphere, by the telescope optics, and by the finite sampling of the image. This "blurring process" can be described in mathematical terms as a convolution. Since the atmospheric conditions are intrinsically variable, this convolution operator is different for each image. Thus to match one image to the PSF of the other one we just need to match the convolution operators. Since a convolution can always be described as a combination of convolutions, one can always transform the first convolution operator to the other one by a convolution with a given kernel. The fundamental purpose of image subtraction is to seek this kernel, and especially to seek the best (optimal) kernel solution.

\subsection{Basics of the Method}

If we need to subtract 2 images, $I_{1}$ and $I_{2}$, assuming $I_{1}$ is the best seeing (reference image), we have to match $I_{1}$ to $I_{2}$. This can be achieved by convolving $I_{1}$ with the proper (least-squares optimal) kernel solution:

$$
\sum_{\text {pixels }}\left[I_{1} \otimes \text { Kernel }-I_{2}\right]^{2} \quad \text { minimal }
$$

Solving Eq. (1) is a full nonlinear problem which requires a very large amount of computing time (Kochanski, Tyson, \& Fisher 1996). A realistic solution to Eq. (1) needs to be much quicker. Such improvement can be done if one notices that the problem can be transformed into a linear least-squares problem, provided the kernel is expanded on a basis of functions:

$$
\operatorname{Kernel}(u, v)=\sum_{i} a_{i} B_{i}(u, v)
$$

Then Eq. (1) becomes:

$$
\sum_{\text {pixels }}\left[\sum_{i} a_{i} I_{1} \otimes B_{i}-I_{2}\right]^{2} \quad \text { minimal }
$$

which is a simple linear least-squares problem. For the basis of functions a Gaussian polynomial expansion has been selected. These functions have a number of interesting properties (see Alard \& Lupton 1998 for more details):

$$
B_{i, j}=e^{-r^{2}} u^{i} v^{j} \text { with } r=\sqrt{u^{2}+v^{2}}
$$

To complete the presentation of the basic image subtraction method, we show that differential background variations between the images can also be handled in a linear way. If we expand the background variations using 2-dimensional polynomials, Eq. (1) can be transformed into the final linear expression:

$$
\sum_{\text {pixels }}+\left[\sum_{i} a_{i} I_{1} \otimes B_{i} \sum_{j} b_{k, l} x^{k} y^{l}-I_{2}\right]^{2} \text { minimal }
$$




\subsection{Spatially Variable Kernel}

Provided the density of objects is large in the image, one can always cut the image into small pieces and make image subtraction in these small areas. In the crowded fields of the microlensing experiments, the Galactic bulge, or the Magellanic clouds, the density of stars is so large that one can make image subtraction in very small area, where the kernel variations are negligible. However, in fields with a low density of objects (for instance, the fields investigated by supernovae surveys), it is not possible to ignore the variations of the kernel across the field. These kernel variations are usually due to the optics of the telescope. A natural solution is to fit these kernel variations by linear expansion of the kernel coefficients:

$$
\operatorname{Kernel}(x, y, u, v)=\sum_{i} a_{i}(x, y) B_{i}(u, v)
$$

with:

$$
a_{i, j}(x, y)=\sum_{i} c_{i} x^{i} d_{j} y^{j}
$$

Although it is obvious that if the spatial variations are expanded, for instance to order 3 , the number of coefficients to evaluate by least squares will be increased by a factor of 10 . Considering that most of the computations are associated with building a square matrix of scalar products between the least-square vectors, the computing time will be increased by a factor close to 100 . Such computing requirement cannot be afforded with the current computers. Fortunately, it is possible to speed up the calculations by a considerable factor if we notice that the matrix for the spatially variable kernel can be deduced from the matrix relevant to the constant kernel with a minimum number of operations. The basic idea is that provided the images are divided into small stamps which have about the size of the kernel, inside these stamps the kernel variations can be neglected, and the scalar products are almost identical to the scalar products for a constant kernel. Thus, the matrix elements of the spatially variable kernel can be deduced from the constant kernel matrix with a minimum number of operations, the fundamental improvement being that the costly scalar products of the vectors need not to be re-calculated each time. In practice, a full solution of the least squares equations with spatially variable kernel does not require more than 20 or $30 \%$ additional cost with respect to constant kernel solutions. For more details see Alard (1999c).

\subsection{Flux Conservation in Spatially Variable Kernels}

In a spatially variable kernel, the sum of the kernels which corresponds to the flux scaling between the images is not necessary constant. This can be an annoying additional source of noise and it is necessary to remove this degree of freedom from the space of kernel solutions. The most straightforward way to do this is to make a least squares fit with constraints by using Lagrange multipliers. However, this method leads to a set of equations which is costly to solve. A better alternative is to recombine the kernel expansion in order that the constant scaling condition be automatically verified. For instance, if we use the following kernel expansion:

$$
\operatorname{Kernel}(x, y, u, v)=a_{0} B_{0}(u, v)+\Sigma_{i=1, N} a_{i}(x, y)\left[B_{i}(u, v)-B_{0}(u, v)\right]
$$




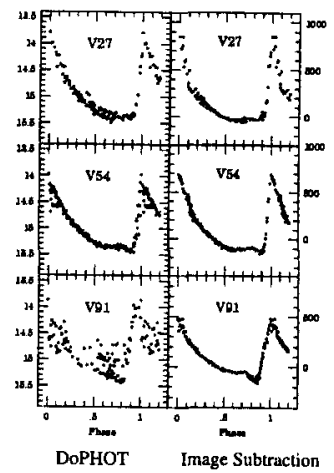

Figure 2. A comparison of the light curves obtained with DoPHOT and image subtraction. Image subtraction can improve the DoPHOT photometry up to a factor of 20 (V91). The variable V54 is a case where the improvement was smaller, but it is still a factor of 2 . V27 is quite typical of the mean improvement for the 26 variables found in common.

provided the $B_{i}$ functions are normalized $\left(\int B(u, v) d u d v=1\right)$, it is very easy to see that $\int \operatorname{Kernel}(x, y, u, v) d u d v$ does not depend any more on the position in the image $(x, y)$.

\section{An Example of Application}

During the J. Kaluzny project of monitoring of globular clusters, a set of 161 frames of M5 were taken with the 1-m telescope in Las Campanas. This data set was processed with the DoPHOT software, allowing the discovery of 26 variables in the central region of M5. The same data set has been re-processed using the image subtraction method described in this article (Olech et al. 1999). Image subtraction revealed a total of 61 variables in the same region where only 26 variables had been found with DoPHOT. A very large improvement in the photometry of the 26 variables that were found by both software programs was also visible. In the mean, image subtraction improves the photometry of M5 by a factor 3 to 4 with respect to DoPHOT, with a maximum improvement close to 20 (see Fig. 2). A comparison of our image subtraction photometry with HST photometry of variables in a small region near the center of M5 (Drissen \& Shara 1998) shows that with only a 1 -m telescope, from the ground a similar accuracy is achieved. The large photometric improvement revealed that the variable V104 previously classified as an eclipsing variable is most likely an RR Lyrae star pulsating in a nonradial mode (see Fig. 3).

\section{Conclusion}

It has been shown that image subtraction is an interesting alternative to classical methods for the analysis of variable stars. Contrary to profile fitting methods, with image subtraction it is possible to find an optimal solution and to approach 


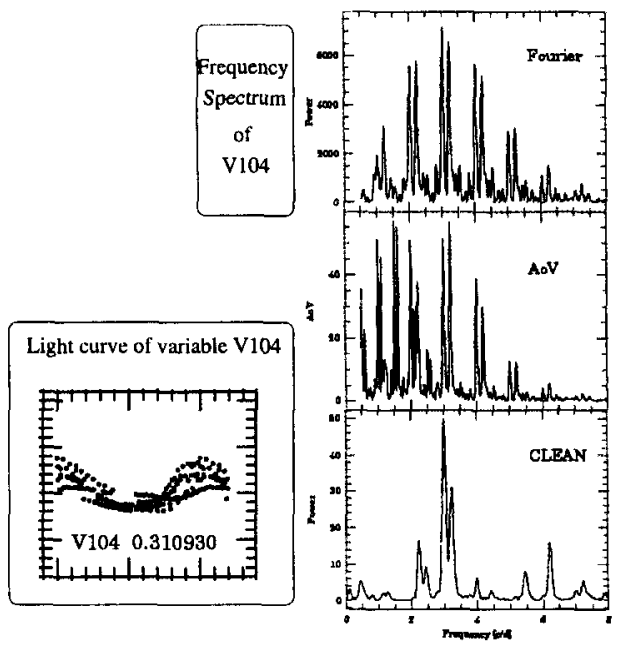

Figure 3. Light curve and frequency spectra for the nonradial pulsator V104. Note the double peak structure of all the spectra, which shows the presence of the nonradial mode.

the theoretical accuracy, even in very dense crowded fields. It is clear that this optimal image subtraction method should be used in any application related to variable stars, or variability in general. To put this in perspective, it is also important to say that image subtraction may change the way we approach the crowded field photometry. More ambitious projects, like monitoring extremely dense regions near the center of globular clusters, or in other galaxies, might be undertaken with quite small telescopes from the ground. It might be possible to investigate some new astrophysical problems with image subtraction which would have been impossible with more classical methods. The only limitation with image subtraction is essentially only the photon noise in the image itself, something which is trivial to estimate.

\section{References}

Alard, C. 1999a, the ISIS package (http://ww.jap.fr/users/alard/package.html)

Alard, C. 1999b, A\&A, 343, 10

Alard, C. 1999c, submitted to A\&A, [astro-ph/9903111]

Alard, C. \& Lupton, R. 1998, ApJ, 503, 1

Drissen, L. \& Shara, M. 1998, AJ, 115, 725

Kochanski, G., Tyson, J., \& Fischer, P. 1996, AJ, 111, 1444

Olech, A., Wozniak, P. R., Alard, D., et al. 1999, MNRAS in press, [astro$\mathrm{ph} / 9905065]$

Pritchard, J. 1999, private communication

Schechter, P., Mateo, M., \& Saha, A. 1993, PASP, 105, 1342

Stetson, P. 1987, PASP, 99, 191 